\title{
A giant trichobezoar extracted by laparoscopic and endoscopic cooperative surgery (LECS)
}

\section{다)(요 $\odot$}

\section{Authors}

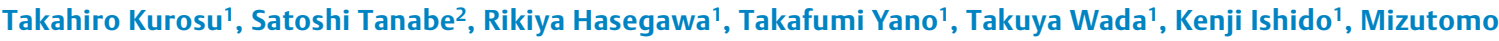
Azuma ${ }^{1}$, Chikatoshi Katada', Wasaburo Koizumi ${ }^{1}$, Hiromitsu Moriya ${ }^{3}$, Keishi Yamashita ${ }^{3}$

Institutions

1 Department of Gastroenterology, Kitasato University School of Medicine, Sagamihara, Kanagawa, Japan

2 Research \& Development Center for New Medical Frontiers, Kitasato University School of Medicine, Sagamihara, Kanagawa, Japan

3 Department of Surgery, Kitasato University School of Medicine, Sagamihara, Kanagawa, Japan

submitted 1.2.2018

accepted after revision 30.5.2018

Bibliography

DOI https://doi.org/10.1055/a-0732-4697 |

Endoscopy International Open 2018; 06: E1413-E1416

(c) Georg Thieme Verlag KG Stuttgart · New York

ISSN 2364-3722

Corresponding author

Takahiro Kurosu, MD, Department of Gastroenterology, Kitasato University School of Medicine, 2-1-1 Asamizodai,
Minami-ku, Sagamihara, Kanagawa 252-0380, Japan

Fax: +81-42-7498690

tk_abc_0521@yahoo.co.jp

\section{ABSTRACT}

A gastric bezoar is a mass that forms in the stomach. A giant gastric bezoar is particularly difficult to treat medically, and surgical therapy is selected. We describe our experience with a patient who had a giant gastric trichobezoar that was extracted by laparoscopic and endoscopic cooperative surgery (LECS) in accordance with the principles of LECS. The patient was a 32-year-old woman who presented at our hospital because of abdominal pain. Upper gastrointestinal endoscopy confirmed the presence of a giant gastric trichobezoar extending from the gastric cardia to the gastric angle. Because endoscopic removal was considered difficult, we extracted the giant gastric trichobezoar by LECS. The concurrent use of endoscopy was considered to allow a gastric bezoar to be extracted more safely and reliably than was previously possible.

\section{Introduction}

A trichobezoar is a rare medical condition that is often difficult to treat medically. In particular, giant gastric bezoars may be treated by highly invasive therapy, such as open surgery [1]. We describe our experience with a patient in whom we successfully extracted a giant gastric trichobezoar in a minimally invasive fashion by laparoscopic and endoscopic cooperative surgery (LECS), performed in accordance with the principles of LECS [2], and we discuss the related literature.

\section{Case report}

A 32-year-old woman presented with abdominal pain. The patient had received oral medical therapy for a diagnosis of schizophrenia since the age of 20 years. Since the diagnosis of schizophrenia, the patient had had a habit of eating her hair. Abdominal pain persisted for several months, and the patient consulted a local physician. A mass was palpated in the abdomen, and computed tomography of the abdomen revealed a giant foreign mass in the stomach. The patient was referred to our hospital for further evaluation and treatment. With the exception of schizophrenia, her medical history was not relevant to the current disorder.

Considering her family history, the patient's mother had a history of gastric cancer. With regard to her lifestyle history, as mentioned above, the patient had a habit of eating her own hair since the diagnosis of schizophrenia. At initial presentation, her height was $150 \mathrm{~cm}$, and body weight was $40 \mathrm{~kg}$. The palpebral conjunctivae were not anemic. The abdomen was flat and soft. A mass was palpated in the lower abdomen. There was no spontaneous pain or tenderness.

Blood biochemical examinations showed no clinically significant abnormal findings. An upper gastrointestinal series ( $\triangleright$ Fig. 1) revealed a giant mass composed of foreign matter, measuring about $290 \times 100 \mathrm{~mm}$, and associated with calcification. The mass occupied a large portion of the stomach. The flow of contrast media into the duodenum was good. Upper 


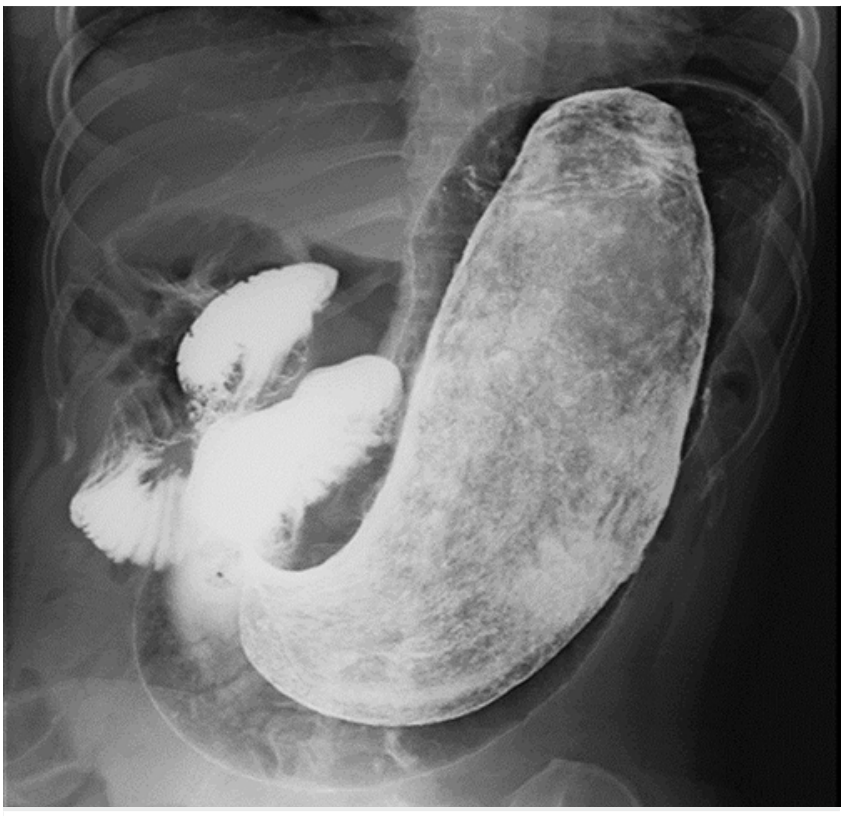

- Fig. 1 Upper gastrointestinal series, showing a giant mass consisting of foreign matter, measuring about $290 \times 100 \mathrm{~mm}$. The mass was associated with calcification and occupied the entire stomach.

gastrointestinal endoscopy ( $\triangleright$ Fig. 2) confirmed the presence of a giant gastric trichobezoar extending from the gastric cardia to the gastric angle.

\section{Clinical course}

A giant gastric trichobezoar was diagnosed on the basis of the findings described above. The giant gastric trichobezoar occupied the entire stomach, and endoscopic removal was considered difficult. We consulted a surgeon in our hospital and decided to perform laparoscopic and endoscopic cooperative surgery (LECS) to remove the gastric bezoar as shown in > Supplementary Video 1. An incision was made in the umbilical region, and a Lap Protector Type S wound retractor (Hakko Medical, Nagano, Japan) was inserted. After the gastric wall had been incised, the Lap Protector $S$ was removed. The gastric wall and skin of the abdominal wound were secured with four supporting sutures, and the Lap Protector $\mathrm{S}$ was reinserted to secure the area between the intra-stomach and the skin after incision of the anterior of the stomach within the wound. An access port (E.Z Access, Hakko Medical) was mounted on the Lap Protector and three $5-\mathrm{mm}$ ports were placed in the E.Z Access to allow laparoscopic and endoscopic examinations. The Lap Protector Type $S$ is a small-type wound retractor that requires a skin incision of 20 to $30 \mathrm{~mm}$, and the E.Z Access is a silicon port for the Lap Protector that makes it possible to insert multiple trocars without air leakage.

The entire gastric trichobezoar was examined by both oral endoscopy (GIF-Q260J, Olympus Corporation, Tokyo, Japan) and laparoscopy, and the mass was sliced thinly using grasping forceps and a scalpel for laparoscopic use that were inserted via the endoscope port. After the gastric trichobezoar had become

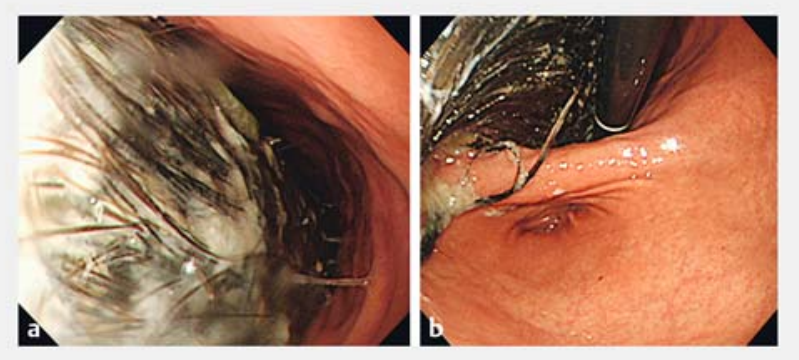

- Fig.2 Upper gastrointestinal endoscopic images. a A giant trichobezoar was present in the stomach. b A giant gastric trichobezoar was confirmed in the region extending from the gastric cardia to the gastric angle.

somewhat smaller, it was pulled out through the Lap Protector $\mathrm{S}$ after removing the $\mathrm{E} \cdot \mathrm{Z}$ Access port. The absence of a remnant bezoar was confirmed, and the incision in the gastric wall was sutured to complete the operation ( $\triangleright$ Fig. $\mathbf{3}$ ).

\section{Discussion}

A gastric bezoar is a foreign body resulting from the accumulation of orally ingested food, hair, and other materials in the stomach. Bezoars can be broadly classified as phytobezoars (fruit, etc.), trichobezoars, drug bezoars, and mixed bezoars according to their main components [3]. Gastric trichobezoars are particularly common in adolescent women who have a habit of eating their hair and should be removed because they carry the risks of gastric ulceration and intestinal obstruction. Treatment options for gastric trichobezoars include medical therapy (non-endoscopic treatments such as Coca-Cola dissolution therapy [4], electrohydraulic lithotripsy [5], laser irradiation [6], extracorporeal shock wave lithotripsy, and endoscopic treatments in which stones are crushed mechanically using a forceps and other instruments) and surgical therapy (open abdominal surgery and laparoscopic surgery to remove gastric bezoars [7-9]). Medical therapy, particularly endoscopic treatment, has the drawbacks of difficulty in securing a working space and treating giant gastric bezoars. On the other hand, surgery carries the risks of scattering the gastric contents in the peritoneal cavity and is a relatively invasive procedure, associated with a large surgical wound and other negative factors.

To overcome the disadvantages of conventional treatments, we simultaneously performed laparoscopic and endoscopic surgery in accordance with the principles of LECS to treat the gastric bezoar. We searched PubMed, and found no study documenting the removal of gastric bezoars by laparoscopic and endoscopic surgery in accordance with the principles of LECS. Only one such patient was described in Gastroenterological Endoscopy, the official journal of the Japan Gastroenterological Endoscopy Society [10]. That report described a gastric bezoar measuring about $100 \mathrm{~mm}$ in diameter that was attempted to be treated by endoscopic lithotripsy and Coca-Cola dissolution therapy, but did not respond. LECS was therefore performed to 


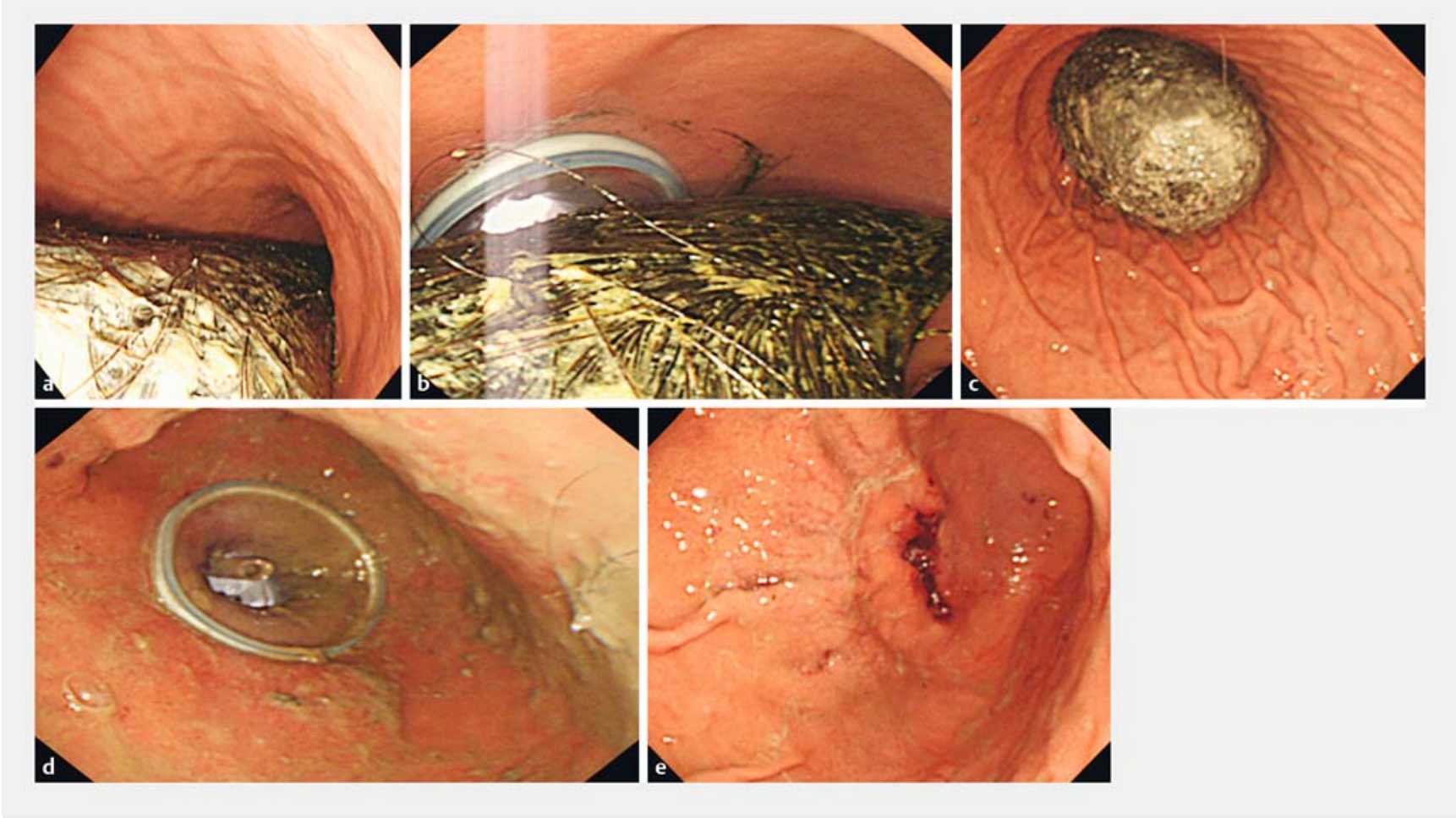

- Fig. 3 Intraoperative endoscopic findings. a A gastric trichobezoar was confirmed on endoscopy. b An E.Z Access was mounted on the Lap Protector, and three 5-mm ports were placed in the E.Z Access to allow laparoscopy and endoscopy. $\mathbf{c}$ The use of endoscopic insufflation allowed the field of surgical vision to be secured. $\mathbf{d}$ The bezoar was resected laparoscopically with the support of endoscopic forceps. e The absence of remnant bezoars was confirmed, and the incision in the gastric wall was sutured to complete the operation.

remove the gastric bezoar. The concurrent use of endoscopy is useful in patients who undergo laparoscopic surgery to treat giant gastric bezoars.

In our case, the use of endoscopy allowed the entire gastric bezoar to be viewed and the supportive use of insufflation and forceps allowed the gastric bezoar to be removed more safely and reliably than was previously possible. LECS is therefore considered to be a useful treatment for giant gastric bezoars and is likely to become a treatment option in the future.

\section{Competing interests}

Drs. Takahiro Kurosu, Satoshi Tanabe, Rikiya Hasegawa, Takafumi Yano, Takuya Wada, Kenji Ishido, Mizutomo Azuma, Chikatoshi Katada, Wasaburo Koizumi, Hiromitsu Moriya, and Keishi Yamashita have no conflicts of interest or financial ties to disclose.

\section{References}

[1] Lamerton A]. Trichobezoar: Two case reports - A new physical sign. Am J Gastroenterol 1984; 79: 354-356

[2] Hiki N, Fukunaga T, Miki A et al. New technique for laparoscopic wedge resection for gastric submucosal tumor: laparoscopy endos- copy cooperative surgery. Jpn J Gastroenterol Surg 2008; 41: 1661 1668

[3] Iwamuro M, Okada H, Matsueda $\mathrm{K}$ et al. Review of the diagnosis and management of gastrointestinal bezoars. World J Gastrointest Endosc 2015; 7: $336-345$

[4] Iwamuro M, Yunoki N, Tomoda J et al. Gastric bezoar treatment by endoscopic fragmentation in combination with Pepsi-Cola ${ }^{\circ}$ administration. Am J Case Rep 2015; 16: 445-448

[5] Kuo JY, Mo LR, Tsai CC et al. Nonoperative treatment of gastric bezoars using electrohydraulic lithotripsy. Endoscopy 1999; 31: $386-$ 388

[6] Grande G, Manno M, Zulli C et al. An alternative endoscopic treatment for massive gastric bezoars: Ho:YAG laser fragmentation. Endoscopy 2016; 48: (Suppl. 01): E217

[7] Kadoya S, Tokuraku M, Harada T et al. A case of bezoar extracted by laparoscopic surgery. J Jpn Surg Assoc 2003; 64: 2741-2744

[8] Ulukent SC, Ozgun YM, Şahbaz NA et al. A modified technique for the laparoscopic management of large gastric bezoars. Saudi Med J 2016; 37: $1022-1024$

[9] Zhang RL, Yang ZL, Fan BG et al. Huge gastric disopyrobezoar: a case report and review of literatures. World J Gastroenterol 2008; 14 : $152-154$

[10] Kuroda K, Fujii M, Shirasaka D et al. A case of a large gastric bezoar extracted by laparoscopy and endoscopy cooperative surgery (LECS). Gastroenterol Endosc 2015; 57: 229-234 (in Japanese with English abstract) 


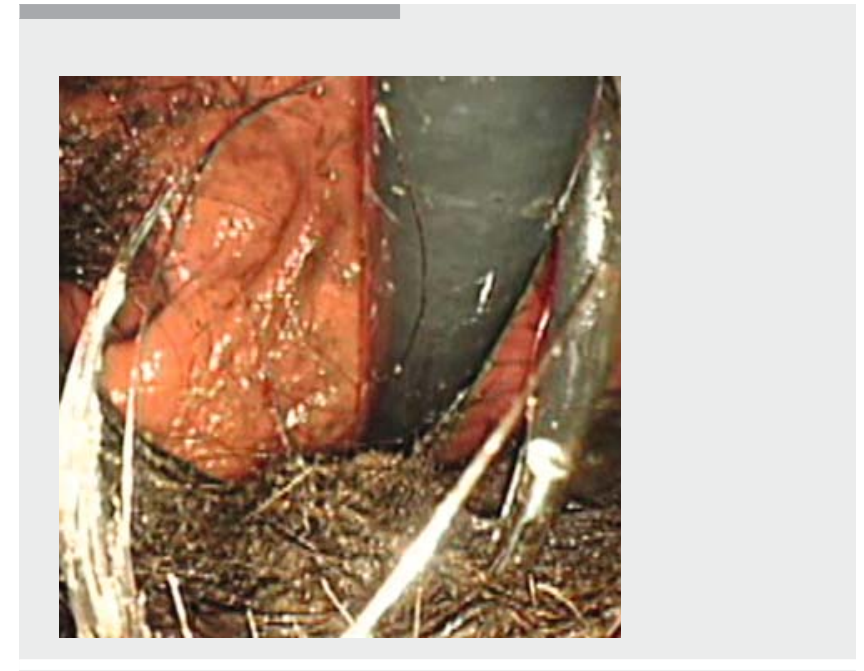

Dupplementary Video 1 Video illustrating laparoscopic and endoscopic cooperative surgery (LECS) to extract a giant gastric trichobezoar. 